\title{
A code to compute the emission of thin accretion disks in non-Kerr space-times and test the nature of black hole candidates
}

\author{
Cosimo Bambi \\ Arnold Sommerfeld Center for Theoretical Physics, \\ Ludwig-Maximilians-Universität München, D-80333 Munich, Germany \\ Center for Field Theory and Particle Physics 83 Department of Physics, \\ Fudan University, 200433 Shanghai, China
}

October 17, 2018

\begin{abstract}
Astrophysical black hole candidates are thought to be the Kerr black holes predicted by General Relativity, but the actual nature of these objects has still to be proven. The analysis of the electromagnetic radiation emitted by a geometrically thin and optically thick accretion disk around a black hole candidate can provide information about the geometry of the space-time around the compact object and it can thus test the Kerr black hole hypothesis. In this paper, I present a code based on a ray-tracing approach and capable of computing some basic properties of thin accretion disks in space-times with deviations from the Kerr background. The code can be used to fit current and future X-ray data of stellar-mass black hole candidates and constrain possible deviations from the Kerr geometry in the spin parameter-deformation parameter plane.
\end{abstract}

Subject headings: accretion, accretion disks — black hole physics — general relativity - X-rays: binaries

\section{Introduction}

The predictions of General Relativity have been confirmed by experiments in Earth's gravitational field (Williams et al. 2004, Everitt et al. 2011), by spacecraft missions in the Solar System (Bertotti et al. 2003), and by accurate radio observations of binary pulsars (Weisberg \& Taylor 2005; Kramer et al. 2006) (for a general review, see e.g. Will (2005)). In all these environments, the gravitational field is weak, in the sense that one can write $g_{t t}=1+\phi$ with $|\phi| \ll 1$. The validity of the theory in the regime of strong gravity, when the 
approximation $|\phi| \ll 1$ breaks down, is instead still unexplored. The ideal laboratory to test strong gravitational fields is the space-time around astrophysical black hole (BH) candidates (Bambi|2011c).

In 4-dimensional General Relativity, uncharged BHs are described by the Kerr solution and are completely specified by two parameters: the mass, $M$, and the spin angular momentum, $J$ (Carter 1971; Robinson 1975). A fundamental limit for a Kerr BH is the bound $|a| \leq M$, where $a=J / M$ is the spin parameter 1 . For $|a|>M$, there is no horizon, and the Kerr metric describes the gravitational field of a naked singularity, which is forbidden by the weak cosmic censorship conjecture (Penrose 1969). On the observational side, there are at least two classes of astrophysical BH candidates (for a review, see e.g. Narayan (2005)): stellar-mass compact objects in X-ray binary systems $\left(M \approx 5-20 M_{\odot}\right)$, and super-massive bodies at the center of every normal galaxy $\left(M \sim 10^{5}-10^{9} M_{\odot}\right)$. The measurement of the mass of the two classes of objects is robust, because obtained by dynamical methods and without any assumption about the nature of these objects. However, this is basically the only solid information we have. We think they are the Kerr BHs predicted by General Relativity because there is no alternative explanation in the framework of conventional physics: stellar-mass BH candidates are too heavy to be neutron star (Kalogera 1996), while at least some of the super-massive objects in galactic nuclei are too heavy, compact, and old to be clusters of non-luminous bodies (Maoz 1998).

The Kerr-nature of astrophysical BH candidates can potentially be tested with already available X-ray data, by extending the two most popular techniques currently used by astronomers to estimate the spin parameter of these objects: the continuum-fitting method (Zhang et al. 1997; Li et al. 2005; McClintock et al. 2011) and the K $\alpha$-iron line analysis (Fabian et al. 1995; Reynolds \& Nowak 2003; Miller 2007). With the continuum-fitting method, one studies the thermal spectrum of geometrically thin and optically thick accretion disks: under the assumption of Kerr background and with independent measurements of the mass of the object, its distance from us, and the inclination angle of the disk, it is possible to infer the spin parameter $a$ and the mass accretion rate $\dot{M}$. The technique can be applied only to stellar-mass BH candidates, as the disk's temperature goes like $M^{-0.25}$ and the spectrum turns out to be in the keV-range for objects in X-ray binary systems, and in the UV for the super-massive bodies at the centers of galaxies. Relaxing the Kerr $\mathrm{BH}$ hypothesis, one can investigate the geometry of the space-time around the BH candidate (Bambi \& Barausse 2011a). With the same spirit, one can analyze the broad K $\alpha$-iron lines observed in both stellar-mass and super-massive BH candidates to test their nature

\footnotetext{
${ }^{1}$ Throughout the paper, I use units in which $G_{\mathrm{N}}=c=1$, unless stated otherwise.
} 
(Johannsen \& Psaltis 2012). Actually, one can usually get only a constraint on a certain combination of the spin parameter and of the deviations from the Kerr background. In order to really test the nature of the compact object, at least two independent measurements are necessary (Bambi 2012b).

With this paper, I am going to present a code designed to compute the thermal emission of a geometrically thin and optically thick accretion disk around a compact object characterized by a mass, an arbitrary value of the spin parameter (that is, no subject to the bound $|a| \leq M$, valid only for Kerr BHs (Bambi 2011a b)), and by one (or more) "deformation parameter(s)", measuring possible deviations from the Kerr geometry. The code is based on a ray-tracing method and takes all the relativistic effects into account, as well as some important astrophysical effects. It thus includes significant improvements with respect to the code used in Bambi \& Barausse (2011a) and can analyze real X-ray data providing the most reliable test we can get with current knowledge. The analysis of the soft X-ray component of specific sources and the corresponding constraints on the spin parameter-deformation parameter plane will be presented in a forthcoming paper (Bambi et al. 2013). The code can also be used to compute other disk's properties, like its direct image and its light curve during an eclipse, which may be observed with future X-ray experiments and can provide additional details about the actual nature of a $\mathrm{BH}$ candidate.

\section{Thermal emission of thin accretion disks}

Geometrically thin and optically thick accretion disks are described by the NovikovThorne model (Novikov \& Thorne 1973; Page \& Thorne 1974), which is the relativistic generalization of the Shakura-Sunyaev model (Shakura \& Sunyaev 1973). Accretion is possible because viscous magnetic/turbulent stresses and radiation transport energy and angular momentum outwards. The model assumes that the disk is on the equatorial plane and that the disk's gas moves on nearly geodesic circular orbits. The time-averaged energy flux emitted from the surface of the disk is (Page \& Thorne 1974)

$$
\mathcal{F}(r)=\frac{\dot{M}}{4 \pi M^{2}} F(r)
$$

where $F(r)$ is the dimensionless function

$$
F(r)=-\frac{\partial_{r} \Omega}{(E-\Omega L)^{2}} \frac{M^{2}}{\sqrt{-G}} \int_{r_{\text {in }}}^{r}(E-\Omega L)\left(\partial_{\rho} L\right) d \rho .
$$

$E, L$, and $\Omega$ are, respectively, the conserved specific energy, the conserved axial-component of the specific angular momentum, and the angular velocity for equatorial circular geodesics 
(in a generic stationary and axisymmetric space-time, these quantities can be computed as described, for instance, in Appendix B of Bambi \& Barausse (2011a)); $G=-\alpha^{2} g_{r r} g_{\phi \phi}$ is the determinant of the near equatorial plane metric, where $\alpha^{2}=g_{t \phi}^{2} / g_{\phi \phi}-g_{t t}$ is the lapse function; $r_{\text {in }}$ is the inner radius of the accretion disk and is assumed to be the radius of the innermost stable circular orbit (ISCO).

Since the disk is in thermal equilibrium, the emission is blackbody-like and we can define an effective temperature $T_{\text {eff }}(r)$ from the relation $\mathcal{F}(r)=\sigma T_{\text {eff }}^{4}$, where $\sigma$ is the StefanBoltzmann constant. Actually, the disk's temperature near the inner edge of the disk can be high, up to $\sim 10^{7} \mathrm{~K}$ for stellar-mass $\mathrm{BH}$ candidates, and non-thermal effects are nonnegligible. That is usually taken into account by introducing the color factor (or hardening factor) $f_{\text {col }}$. The color temperature is $T_{\text {col }}(r)=f_{\text {col }} T_{\text {eff }}$ and the local specific intensity of the radiation emitted by the disk is

$$
I_{\mathrm{e}}\left(\nu_{\mathrm{e}}\right)=\frac{2 h \nu_{\mathrm{e}}^{3}}{c^{2}} \frac{1}{f_{\text {col }}^{4}} \frac{\Upsilon}{\exp \left(\frac{h \nu_{\mathrm{e}}}{k_{\mathrm{B}} T_{\mathrm{col}}}\right)-1},
$$

where $\nu_{\mathrm{e}}$ is the photon frequency, $h$ is the Planck's constant, $c$ is the speed of light, $k_{\mathrm{B}}$ is the Boltzmann constant, and $\Upsilon$ is a function of the angle between the wavevector of the photon emitted by the disk and the normal of the disk surface, say $\xi$. The two most common options are $\Upsilon=1$ (isotropic emission) and $\Upsilon=\frac{1}{2}+\frac{3}{4} \cos \xi$ (limb-darkened emission).

\subsection{Calculation method}

The calculation of the thermal spectrum of a thin accretion disk has been extensively discussed in the literature; see e.g. Li et al. (2005) and references therein. The spectrum can be conveniently written in terms of the photon flux number density as measured by a distant observer, $N_{E_{\text {obs }}}$. A quite common approximation in the literature is to neglect the effect of light bending, which is good for small disk's inclination angles (face-on disks). In this case, $N_{E_{\mathrm{obs}}}$ is:

$$
\begin{aligned}
N_{E_{\mathrm{obs}}} & =\frac{1}{E_{\mathrm{obs}}} \int I_{\mathrm{obs}}(\nu) d \Omega_{\mathrm{obs}}= \\
& =\frac{1}{E_{\mathrm{obs}}} \int g^{3} I_{\mathrm{e}}\left(\nu_{\mathrm{e}}\right) d \Omega_{\mathrm{obs}}= \\
& =A_{1}\left(\frac{E_{\mathrm{obs}}}{\mathrm{keV}}\right)^{2} \cos i \int_{\text {disk }} \frac{1}{M^{2}} \frac{\Upsilon \sqrt{-G} d r d \phi}{\exp \left[\frac{A_{2}}{g F^{1 / 4}}\left(\frac{E_{\mathrm{obs}}}{\mathrm{keV}}\right)\right]-1},
\end{aligned}
$$

where $I_{\mathrm{obs}}, E_{\mathrm{obs}}$, and $\nu$ are, respectively, the specific intensity of the radiation, the photon energy, and the photon frequency measured by the distant observer. $d \Omega_{\text {obs }} \approx \cos i \sqrt{-G} d r d \phi / D^{2}$ 


\begin{tabular}{ll}
\hline \hline Geometry of the background & Johannsen-Psaltis space-time with three parame- \\
& ters $($ mass $M$, spin parameter $a$, deformation pa- \\
& rameter $\left.\epsilon_{3}\right)$. When $\epsilon_{3}=0$, the geometry of the \\
& space-time reduces exactly to the Kerr solution. \\
& No restrictions on the values of $a / M$ and $\epsilon_{3}$ \\
& All relativistic effects are included. Ray-tracing \\
& technique used \\
Relativistic effects & Not included \\
Self-irradiation & Not included \\
Non-zero torque at $r_{\text {in }}$ & Not included \\
Indirect images & Constant. Set by the user \\
Color factor $f_{\text {col }}$ & Isotropic or limb-darkened \\
Radiation emission $\Upsilon$ &
\end{tabular}

Table 1: Basic features of the code to compute the observed spectrum of the disk. Indirect images are the images formed by null geodesics penetrating the equatorial plane inside $r_{\text {in }}$.

\begin{tabular}{ccccc}
\hline \hline$a / M$ & $\epsilon_{3}$ & $r_{\mathrm{H}} / M$ & $r_{\mathrm{ISCO}} / M$ & ISCO \\
\hline \hline 0.0 & 0 & 2 & 6 & MRS \\
0.5 & 0 & 1.8660 & 4.2330 & MRS \\
0.9 & 0 & 1.4359 & 2.3209 & MRS \\
0.999 & 0 & 1.0447 & 1.1818 & MRS \\
\hline 0.8 & 2 & $1.6(\theta=0, \pi)$ & 1.9048 & MVS \\
0.9 & 1 & $1.4359(\theta=0, \pi)$ & 1.7499 & MVS \\
\hline 0.9 & -1 & $1.6176(\theta=\pi / 2)$ & 3.2304 & MRS \\
1.1 & -0.5 & $1.2801(\theta=\pi / 2)$ & 2.1139 & MRS \\
1.2 & -1 & $1.3614(\theta=\pi / 2)$ & 2.5036 & MRS \\
\hline \hline
\end{tabular}

Table 2: Properties of the backgrounds shown in Figs. 1.7: spin parameter a (first column), deformation parameter $\epsilon_{3}$ (second column), radius of the event horizon $r_{\mathrm{H}}$ (third column), radius of the ISCO $r_{\text {ISCO }}$ (fourth column), and stability of the ISCO (MRS/MVS = Marginally Radially/Vertically Stable; fifth column). When $\epsilon_{3} \neq 0$, the radius of the event horizon in general depends on the angle $\theta$ and the topology of the event horizon may be non-trivial (see Bambi \& Modesto (2011)). For the cases $\left(a / M, \epsilon_{3}\right)=(0.8,2)$ and $(0.9,1)$, there is no event horizon on the equatorial plane $\theta=\pi / 2$. 

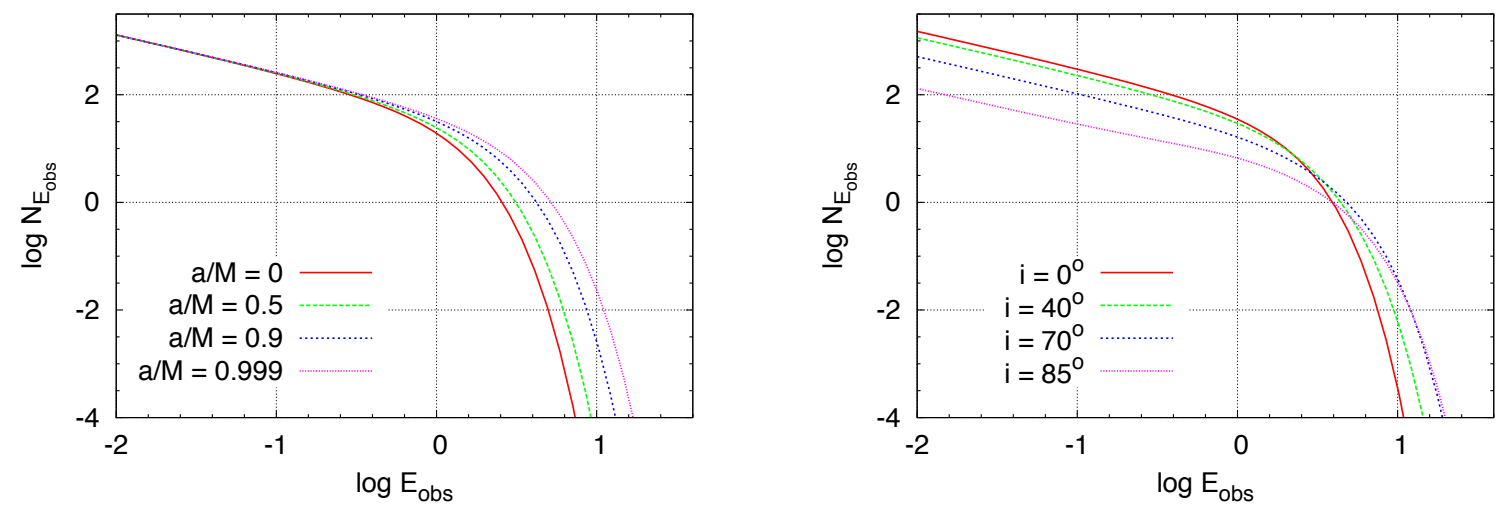

Fig. 1.- Observed thermal spectrum of a thin accretion disk in Kerr space-time. Left panel: $a / M=0,0.5,0.9$, and 0.999 and viewing angle $i=30^{\circ}$. Right panel: $a / M=0.9$ and viewing angle $i=0^{\circ}, 40^{\circ}, 70^{\circ}$, and $85^{\circ}$. The other parameters are $M=10 M_{\odot}, \dot{M}=10^{19} \mathrm{~g} \mathrm{~s}^{-1}$, $D=10 \mathrm{kpc}, f_{\text {col }}=1$, and $\Upsilon=1$. These two plots should be compared with the two panels of Fig. 5 in Li et al. (2005). Flux density $N_{E_{\text {obs }}}$ in $\gamma \mathrm{keV}^{-1} \mathrm{~cm}^{-2} \mathrm{~s}^{-1}$; photon energy $E_{\text {obs }}$ in $\mathrm{keV}$.
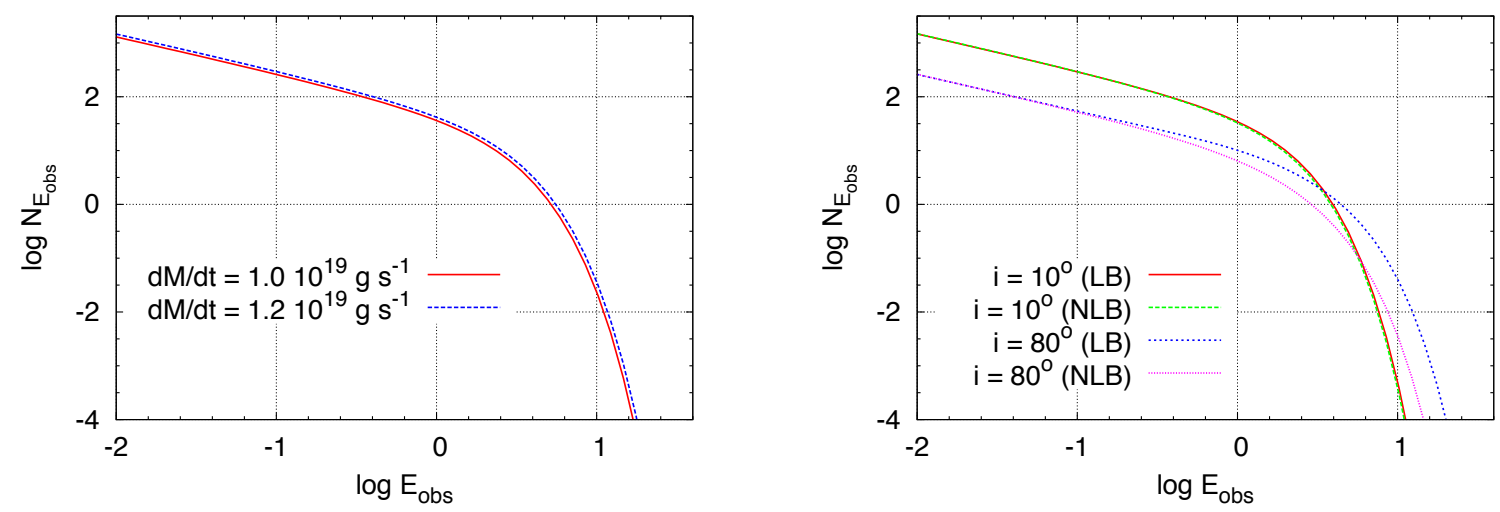

Fig. 2.- Left panel: Kerr space-time with $\dot{M}=1.0 \cdot 10^{19} \mathrm{~g} \mathrm{~s}^{-1}$ and $1.2 \cdot 10^{19} \mathrm{~g} \mathrm{~s}^{-1}$. The other parameters are $M=10 M_{\odot}, a / M=0.999, i=30^{\circ}, D=10 \mathrm{kpc}, f_{\text {col }}=1$, and $\Upsilon=1$. The outer radius of the accretion disk is at $r_{\text {out }}=10^{5} \mathrm{M}$. Right panel: Effect of light bending on the observed spectrum in Kerr space-time for a viewing angle $i=10^{\circ}$ and $80^{\circ} . \mathrm{LB} / \mathrm{NLB}=$ light bending $/$ no light bending. The other parameters are $M=10 M_{\odot}$, $a / M=0.9, \dot{M}=1.0 \cdot 10^{19} \mathrm{~g} \mathrm{~s}^{-1}, D=10 \mathrm{kpc}, f_{\text {col }}=1$, and $\Upsilon=1$. The outer radius of the accretion disk is at $r_{\text {out }}=10^{5} \mathrm{M}$. Flux density $N_{E_{\mathrm{obs}}}$ in $\gamma \mathrm{keV}^{-1} \mathrm{~cm}^{-2} \mathrm{~s}^{-1}$; photon energy $E_{\text {obs }}$ in $\mathrm{keV}$. 
is the element of the solid angle subtended by the image of the disk on the observer's sky, $i$ is the viewing angle of the observer, and $D$ is the distance of the source. $g$ is the redshift factor

$$
g=\frac{E_{\mathrm{obs}}}{E_{\mathrm{e}}}=\frac{\nu}{\nu_{\mathrm{e}}}=\frac{k_{\alpha} u_{\mathrm{obs}}^{\alpha}}{k_{\beta} u_{\mathrm{e}}^{\beta}}
$$

where $E_{\mathrm{e}}=h \nu_{\mathrm{e}}, k^{\alpha}$ is the 4-momentum of the photon, $u_{\mathrm{obs}}^{\alpha}=(-1,0,0,0)$ is the 4-velocity of the distant observer, and $u_{\mathrm{e}}^{\alpha}=\left(u_{\mathrm{e}}^{t}, 0,0, \Omega u_{\mathrm{e}}^{t}\right)$ is the 4 -velocity of the emitter. $I_{\mathrm{e}}\left(\nu_{\mathrm{e}}\right) / \nu_{\mathrm{e}}^{3}=$ $I_{\text {obs }}\left(\nu_{\text {obs }}\right) / \nu^{3}$ follows from the Liouville's theorem. $A_{1}$ and $A_{2}$ are given by (for the sake of clarity, here I show explicitly $G_{\mathrm{N}}$ and $c$ )

$$
\begin{aligned}
A_{1} & =\frac{2(\mathrm{keV})^{2}}{f_{\mathrm{col}}^{4}}\left(\frac{G_{\mathrm{N}} M}{c^{3} h D}\right)^{2}= \\
& =\frac{0.07205}{f_{\mathrm{col}}^{4}}\left(\frac{M}{M_{\odot}}\right)^{2}\left(\frac{\mathrm{kpc}}{D}\right)^{2} \gamma \mathrm{keV}^{-1} \mathrm{~cm}^{-2} \mathrm{~s}^{-1} \\
A_{2} & =\left(\frac{\mathrm{keV}}{k_{\mathrm{B}} f_{\mathrm{col}}}\right)\left(\frac{G_{\mathrm{N}} M}{c^{3}}\right)^{1 / 2}\left(\frac{4 \pi \sigma}{\dot{M}}\right)^{1 / 4}= \\
& =\frac{0.1331}{f_{\mathrm{col}}}\left(\frac{10^{18} \mathrm{~g} \mathrm{~s}^{-1}}{\dot{M}}\right)^{1 / 4}\left(\frac{M}{M_{\odot}}\right)^{1 / 2}
\end{aligned}
$$

Using the normalization condition $g_{\mu \nu} u_{\mathrm{e}}^{\mu} u_{\mathrm{e}}^{\nu}=-1$, one finds

$$
u_{\mathrm{e}}^{t}=-\frac{1}{\sqrt{-g_{t t}-2 g_{t \phi} \Omega-g_{\phi \phi} \Omega^{2}}},
$$

and therefore

$$
g=\frac{\sqrt{-g_{t t}-2 g_{t \phi} \Omega-g_{\phi \phi} \Omega^{2}}}{1+\lambda \Omega}
$$

where $\lambda=k_{\phi} / k_{t}$ is a constant of the motion along the photon path. Neglecting the effect of light bending, $\lambda=r \sin \phi \sin i$, where $r$ and $\phi$ are the coordinates on the equatorial plane of the emitter. Doppler boosting, gravitational redshift, and frame dragging are entirely encoded in the redshift factor $g$.

The effect of light bending can be included by using a ray-tracing approach. The initial conditions $\left(t_{0}, r_{0}, \theta_{0}, \phi_{0}\right)$ for the photon with Cartesian coordinates $(X, Y)$ on the image plane 
of the distant observer are given by (Johannsen \& Psaltis 2010$)$

$$
\begin{aligned}
t_{0} & =0 \\
r_{0} & =\sqrt{X^{2}+Y^{2}+D^{2}} \\
\theta_{0} & =\arccos \frac{Y \sin i+D \cos i}{\sqrt{X^{2}+Y^{2}+D^{2}}}, \\
\phi_{0} & =\arctan \frac{X}{D \sin i-Y \cos i} .
\end{aligned}
$$

As the initial 3-momentum $\mathbf{k}_{\mathbf{0}}$ must be perpendicular to the plane of the image of the observer, the initial conditions for the 4-momentum of the photon are (Johannsen \& Psaltis 2010)

$$
\begin{aligned}
k_{0}^{r} & =-\frac{D}{\sqrt{X^{2}+Y^{2}+D^{2}}}\left|\mathbf{k}_{\mathbf{0}}\right|, \\
k_{0}^{\theta} & =\frac{\cos i-D \frac{Y \sin i+D \cos i}{X^{2}+Y^{2}+D^{2}}}{\sqrt{X^{2}+(D \sin i-Y \cos i)^{2}}}\left|\mathbf{k}_{\mathbf{0}}\right|, \\
k_{0}^{\phi} & =\frac{X \sin i}{X^{2}+(D \sin i-Y \cos i)^{2}}\left|\mathbf{k}_{\mathbf{0}}\right|, \\
k_{0}^{t} & =\sqrt{\left(k_{0}^{r}\right)^{2}+r_{0}^{2}\left(k_{0}^{\theta}\right)^{2}+r_{0}^{2} \sin ^{2} \theta_{0}\left(k_{0}^{\phi}\right)^{2}} .
\end{aligned}
$$

In the numerical calculation, the observer is located at $D=10^{6} \mathrm{M}$, which is far enough to assume that the background geometry is flat and therefore $k_{0}^{t}$ can be inferred from the condition $g_{\mu \nu} k^{\mu} k^{\nu}=0$ with the metric tensor of a flat space-time. The photon trajectory is numerically integrated backwards in time to the point of the photon emission on the accretion disk: in this way, we get the radial coordinate $r_{\mathrm{e}}$ at which the photon was emitted and the angle $\xi$ between the wavevector of the photon and the normal of the disk surface (necessary to compute $\Upsilon$ ). Now $g=g(X, Y)$, as in Eq. (8) everything depends on $r_{\mathrm{e}}$, except $\lambda$ that can be evaluated from the photon initial conditions $\left(\lambda=k_{\phi} / k_{t}=r_{0} \sin \theta_{0} k_{0}^{\phi} / k_{0}^{t}\right)$. The observer's sky is divided into a number of small elements and the ray-tracing procedure provides the observed flux density from each element; summing up all the elements, we get the total observed flux density of the disk. In the case of Kerr background, one can actually exploit the special properties of the Kerr solution and solve a simplified set of differential equations. That is not possible in a generic non-Kerr background and so the code solves the second-order photon geodesic equations of the space-time, by using the fourth-order Runge-Kutta-Nyström method (Lund et al. 2009). The photon flux number density is given 
by

$$
\begin{aligned}
N_{E_{\mathrm{obs}}} & =\frac{1}{E_{\mathrm{obs}}} \int I_{\mathrm{obs}}(\nu) d \Omega_{\mathrm{obs}}=\frac{1}{E_{\mathrm{obs}}} \int g^{3} I_{\mathrm{e}}\left(\nu_{\mathrm{e}}\right) d \Omega_{\mathrm{obs}}= \\
& =A_{1}\left(\frac{E_{\mathrm{obs}}}{\mathrm{keV}}\right)^{2} \int \frac{1}{M^{2}} \frac{\Upsilon d X d Y}{\exp \left[\frac{A_{2}}{g F^{1 / 4}}\left(\frac{E_{\mathrm{obs}}}{\mathrm{keV}}\right)\right]-1},
\end{aligned}
$$

where $X$ and $Y$ are the coordinates of the position of the photon on the sky, as seen by the distant observer; that is, $d \Omega_{\mathrm{obs}}=d X d Y / D^{2}$. The basic features of the code are outlined in Tab. 1.

The results of the code in the Kerr background are summarized in Figs. 1 and 2, while Tab. 2 shows some fundamental properties of these space-times. The two panels of Fig. 1 should be compared with the ones in Fig. 5 of Li et al. (2005). The agreement between the two sets of spectra is very good, except for the case $a / M=0.999$ and inclination angle $i=30^{\circ}$, for which the difference is however small. The discrepancy is due to the fact that the present code does not include the effect of self-irradiation of the disk. As discussed in $\mathrm{Li}$ et al. (2005), the effect of self-irradiation of the disk, as well as a possible non-zero torque at the inner edge of the disk (also not included here, see Tab. 1), can be ignored in the analysis of observational data, because they can be absorbed by adjusting the mass accretion rate and the spectral color factor of a zero torque model without returning radiation. This is indeed our case, as shown in the left panel of Fig. 2, where we can see the spectra of a Kerr $\mathrm{BH}$ with $a / M=0.999$ and $i=30^{\circ}$, respectively for $\dot{M}=1.0 \cdot 10^{19}$ and $1.2 \cdot 10^{19} \mathrm{~g} \mathrm{~s}^{-1}$. The spectrum with higher mass accretion rate and without self-irradiation of the disk is in agreement with the one calculated in Li et al. (2005) for $\dot{M}=1.0 \cdot 10^{19} \mathrm{~g} \mathrm{~s}^{-1}$ and with selfirradiation ${ }^{2}$. The effect of light bending can be seen in the right panel in Fig. 2, which shows the observed spectra as computed respectively from Eq. (4) and Eq. (17). The difference in the observed spectra is significant for large inclination angles (edge-on disks) and negligible in the opposite case, when the angle between the observer and the symmetry axes of the system is small (face-on disks). In all these spectra, the inner edge of the disk is at the ISCO radius (Novikov-Thorne model), while the outer edge is assumed at $r_{\text {out }}=10^{5} \mathrm{M}$. The latter is large enough that for the energy range shown in Figs. 1, 4 is equivalent to an infinite value.

${ }^{2}$ With the standard approach in a Kerr background, one has two parameters, $a$ and $\dot{M}$, to be determined by fitting the soft X-ray component with the theoretical prediction of the thermal spectrum of a thin disk. Li et al. (2005) shows that disk's self-irradiation and small non-zero torque at the ISCO can be neglected without affecting the measurement of $a$ : one just gets a slightly different $\dot{M}$, which has not physical implications. As the physical mechanisms responsible to these effects are not peculiar of the Kerr geometry, the same conclusion should be true for metrics with deviations from Kerr. 
The effect of $r_{\text {out }}$ is indeed to introduce a different slope of the spectrum at low energies, see e.g. Fig. 4 in Bambi \& Barausse (2011a).

\subsection{Non-Kerr space-times}

The Kerr-nature of astrophysical BH candidates can be tested by studying the thermal spectrum of the accretion disk without the assumption of the Kerr background. The basic idea is to consider a more general space-time, in which the compact object is characterized by a mass $M$, a spin parameter $a$, and a deformation parameter which measures possible deviation from the Kerr geometry. When the deformation parameter is set to zero, we have to recover exactly the Kerr solution. We can then compare the theoretical predictions with observational data: if the latter demand a zero deformation parameter, the Kerr $\mathrm{BH}$ hypothesis is verified; otherwise, we may conclude that the object is not a Kerr BH.

Let us note that this approach can be used just to check if the geometry of the spacetime is described by the Kerr solution, but it cannot really investigate the actual nature of the compact object or the exact deviations from the Kerr background. The analysis of the disk's spectrum can only probe the geometry of the space-time till the ISCO radius; we

have no information about the geometry at smaller radii and about the surface/horizon of the BH candidate. Current (and near-future) data are not so good to map the space-time and a single deformation parameter is used to figure out if the gravitational force is stronger or weaker than the one around a Kerr BH with the same mass and spin. Actually, the typical situation is even worse and we can infer only one parameter: if we assume the Kerr background, we find the spin $a$, if we have also a deformation parameter, we constrain some combination of $a$ and of the deformation parameter. However, this degeneracy can be solved with an additional measurement (Bambi 2012b $\mathrm{c}$ d).

When the computation of the properties of the thermal emission depends on the geometry of the background, the code calls the function metric. It is thus easy to change the space-time. For the time being, the code uses the Johannsen-Psaltis (JP) metric, which was explicitly proposed in Johannsen \& Psaltis (2011) to test the geometry around BH candidates. In Boyer-Lindquist coordinates, the metric is given by the line element (Johannsen \& Psaltis 2011)

$$
\begin{aligned}
d s^{2}= & -\left(1-\frac{2 M r}{\Sigma}\right)(1+h) d t^{2}-\frac{4 a M r \sin ^{2} \theta}{\Sigma}(1+h) d t d \phi+\frac{\Sigma(1+h)}{\Delta+a^{2} h \sin ^{2} \theta} d r^{2}+ \\
& +\Sigma d \theta^{2}+\left[\sin ^{2} \theta\left(r^{2}+a^{2}+\frac{2 a^{2} M r \sin ^{2} \theta}{\Sigma}\right)+\frac{a^{2}(\Sigma+2 M r) \sin ^{4} \theta}{\Sigma} h\right] d \phi^{2}
\end{aligned}
$$


where

$$
\begin{aligned}
\Sigma & =r^{2}+a^{2} \cos ^{2} \theta \\
\Delta & =r^{2}-2 M r+a^{2} \\
h & =\sum_{k=0}^{\infty}\left(\epsilon_{2 k}+\frac{M r}{\Sigma} \epsilon_{2 k+1}\right)\left(\frac{M^{2}}{\Sigma}\right)^{k} .
\end{aligned}
$$

This metric has an infinite number of deformation parameters $\epsilon_{i}$ and the Kerr solution is recovered when all the deformation parameters vanish. However, in order to reproduce the correct Newtonian limit, we have to impose $\epsilon_{0}=\epsilon_{1}=0$, while $\epsilon_{2}$ is strongly constrained by Solar System experiments. As done in other papers, it is usually sufficient to restrict the attention to the deformation parameter $\epsilon_{3}$ and set to zero all the others.

The properties of JP BHs with $M, a$, and $\epsilon_{3}$ have been quite discussed in the recent literature (Bambi 2011d, 2012a b c d; Bambi \& Modesto 2011; Bambi et al. 2012; Johannsen \& Psaltis 2012; Chen \& Jing 2012; Liu et al. 2012; Krawczynski 2012). One can note that these objects may have properties fundamentally different from the ones expected for Kerr BHs. For instance, when the gravitational force on the equatorial plane turns out to be stronger/weaker than the Kerr case (this corresponds to $\epsilon_{3}<0 / \epsilon_{3}>0$ ), the maximum value of $a / M$ can presumably be larger/smaller than 1 , and increases/decreases as $\epsilon_{3}$ decreases/increases (Bambi 2011d). Another important feature to bear in mind is that the ISCO may not be determined by the orbital stability along the radial direction, as in Kerr, but it may be marginally vertically stable. The ISCO radius should thus be computed as described in Bambi \& Barausse (2011a b). The event horizon of JP BHs has been discussed in Bambi \& Modesto (2011) and the radius $r_{\mathrm{H}}$ can be found from ${ }^{3}$

$$
\Delta+a^{2} h \sin ^{2} \theta=0 .
$$

For non-vanishing $a$ and $h$, the radius $r_{\mathrm{H}}$ is a function of the angle $\theta$. It is also possible to show that the topology of the event horizon of these BHs may be non-trivial and that BHs

\footnotetext{
${ }^{3}$ Let us note that there are a few definitions of horizon. Here we are talking about the event horizon, i.e. a boundary in the space-time beyond which events cannot affect an outside observer. In a stationary spacetime, the event horizon is also an apparent horizon, which is a surface of zero expansion for a congruence of outgoing null geodesics orthogonal to the surface. This means that at the apparent horizon null geodesics must have $d r / d t=0$, which implies $g^{r r}=0$, see e.g. Poisson (2004). Eq. (20) follows from $g^{r r}=0$ in the case of the JP metric. The horizon relevant for the black hole thermodynamics is instead the Killing horizon, which is a null hyper-surface on which there is a null Killing vector field. For the metric in (18), the Killing horizon is defined by $g_{t t} g_{\phi \phi}-g_{t \phi}^{2}=0$. When the Hawking's rigidity theorem can be applied (like in the Kerr space-time), the event horizon and the Killing horizon coincide (Hawking 1972). However, in general that is not true.
} 
with a topologically non-trivial event horizon may be created by astrophysical processes like the accretion from disk (Bambi \& Modesto 2011). The fundamental properties of the JP space-times shown in Figs. 3.7 are reported in Tab. 2 .

Examples of the observed spectrum of thin accretion disks in the JP background are shown in Fig. 3, for $a / M=0.8$ and $\epsilon_{3}=2$ (left panel) and for $a / M=1.2$ and $\epsilon_{3}=-1$ (right panel). Fig. 3 shows the effect of light bending in the JP background. As in the Kerr case, the effect is more and more important as the disk's inclination angle increases and the observer approaches the equatorial plane of the system. Fig. 4 shows instead the effect on the observed spectrum of the properties of the emission of the accretion disk. It compares the cases of isotropic $(\Upsilon=1)$ and limb-darkened emission $\left(\Upsilon=\frac{1}{2}+\frac{3}{4} \cos \xi, \xi\right.$ being the angle between the wavevector of the photon emitted by the disk and the normal of the disk surface). The left panel of Fig. 4 can be compared with Fig. 9 of Li et al. (2005). As noticed in Li et al. (2005), for edge-on disks the effect of limb-darkening cannot be absorbed by a redefinition of $\dot{M}$ and $f_{\text {col }}$; the spectrum has indeed a more pronounced hump before the exponential cut-off. The case of non-Kerr background does not introduce any new qualitatively different feature with respect to the Kerr case already discussed in the literature.

\section{Other observable features}

In the previous sections, I showed how the code can compute the thermal spectrum of a geometrically thin and optically thick accretion disk in a generic space-time, comparing the results for the Kerr case with the ones presented in Li et al. (2005). While even the continuum-fitting method is not immune to criticism, this approach to test the nature of astrophysical $\mathrm{BH}$ candidates is likely the best we can do with current observational data and theoretical knowledge (McClintock et al. 2011). Constraints on the spin parameterdeformation parameter plane obtained with the code by analyzing the data of specific sources in the high-soft state will be presented in a companion paper (Bambi et al. 2013). In this section, I am going to show some simple extensions of the code: the computation of the direct image of the disk and the prediction of the light curve during an eclipse. Current facilities cannot observe these features, but future X-ray experiments may do the job and provide additional information about the geometry of the space-time around $\mathrm{BH}$ candidates. 

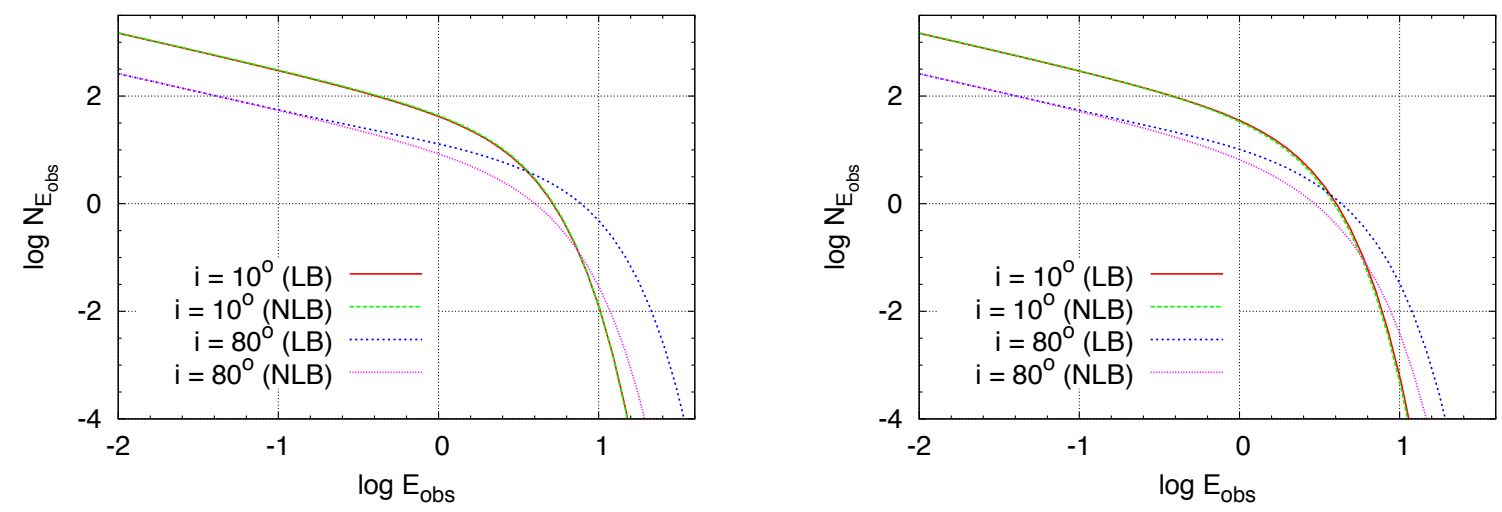

Fig. 3.- Effect of light bending on the observed spectrum in Johannsen-Psaltis space-time. Left panel: $a / M=0.8, \epsilon_{3}=2$, and viewing angle $i=10^{\circ}$ and $80^{\circ}$. Right panel: $a / M=1.2$, $\epsilon_{3}=-1$, and viewing angle $i=10^{\circ}$ and $80^{\circ}$. LB/NLB $=$ light bending $/$ no light bending. The other parameters are $M=10 M_{\odot}, \dot{M}=10^{19} \mathrm{~g} \mathrm{~s}^{-1}, D=10 \mathrm{kpc}, f_{\text {col }}=1$, and $\Upsilon=1$. The outer radius of the accretion disk is at $r_{\text {out }}=10^{5} M$. Flux density $N_{E_{\text {obs }}}$ in $\gamma \mathrm{keV}^{-1}$ $\mathrm{cm}^{-2} \mathrm{~s}^{-1}$; photon energy $E_{\text {obs }}$ in $\mathrm{keV}$.
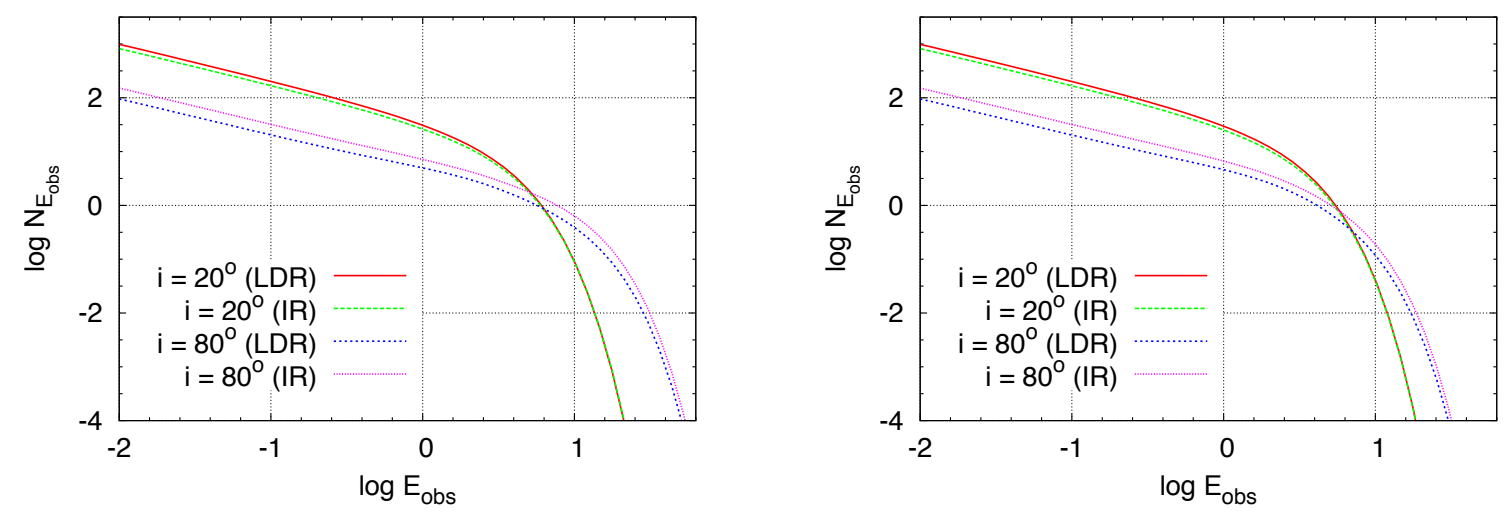

Fig. 4.- Effect of limb-darkened emission on the observed spectrum. Left panel: Kerr space-time with $a / M=0.999$ and viewing angle $i=20^{\circ}$ and $80^{\circ}$. Right panel: JohannsenPsaltis space-time with $a / M=1.1, \epsilon_{3}=-0.5$, and viewing angle $i=20^{\circ}$ and $80^{\circ}$. The other parameters are $M=10 M_{\odot}, \dot{M}=10^{19} \mathrm{~g} \mathrm{~s}^{-1}, D=10 \mathrm{kpc}$, and $f_{\text {col }}=1.5$. LDR $/ \mathrm{IR}=$ limb-darkened/isotropic radiation. The outer radius of the accretion disk is at $r_{\text {out }}=10^{5} M$. The left panel can be compared with Fig. 9 of Li et al. (2005). Flux density $N_{E_{\text {obs }}}$ in $\gamma \mathrm{keV}^{-1}$ $\mathrm{cm}^{-2} \mathrm{~s}^{-1}$; photon energy $E_{\text {obs }}$ in $\mathrm{keV}$. 

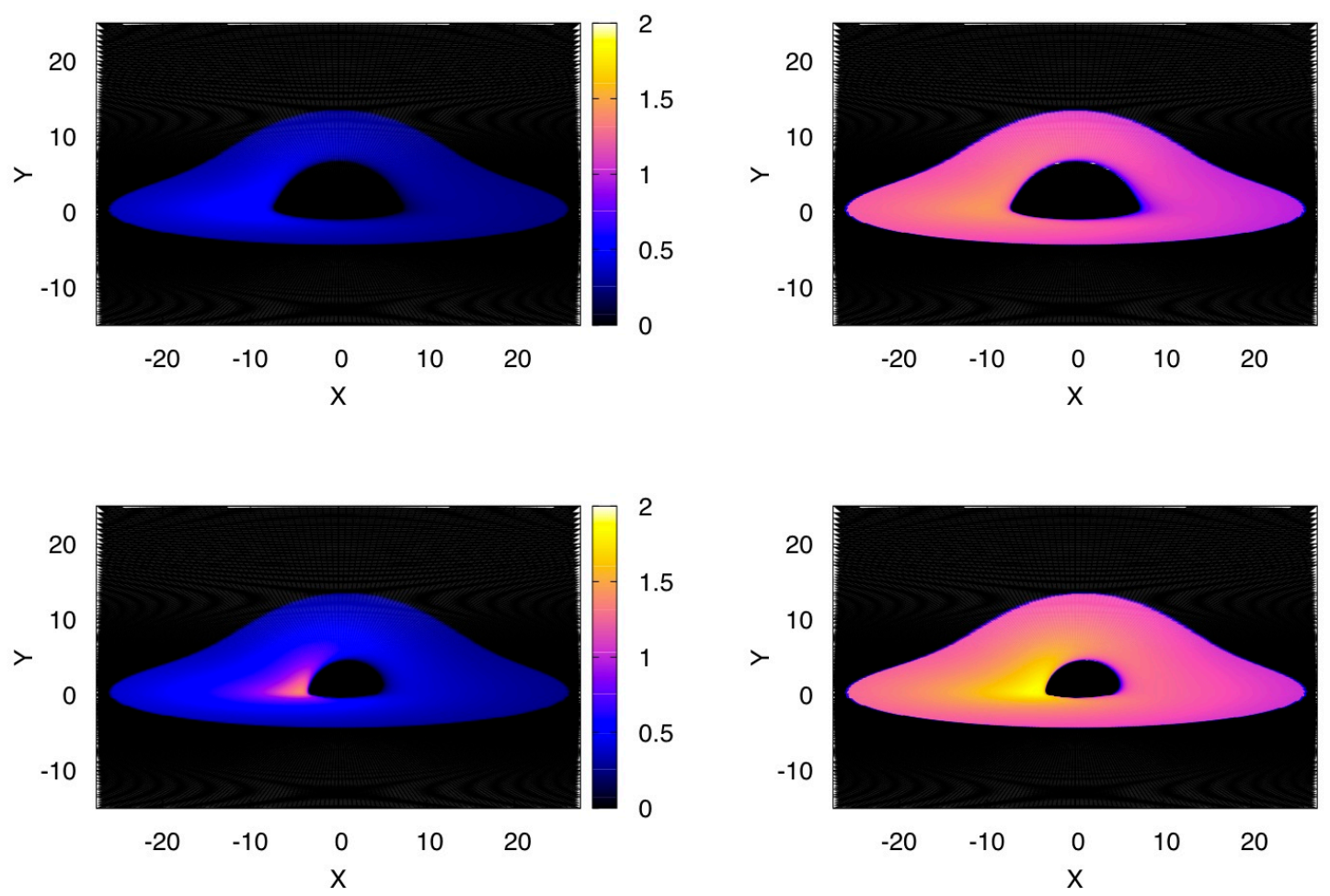

Fig. 5.- Direct image of the accretion disk. Observed blackbody temperature $T_{\text {obs }}$ (left panels) and observed flux $\mathcal{F}_{\text {obs }}$ (right panels) in Kerr space-time with spin parameter $a / M=0$ (top panels) and 0.9 (bottom panels). The other parameters are $M=10 M_{\odot}, \dot{M}=10^{18} \mathrm{~g} \mathrm{~s}^{-1}$, $i=80^{\circ}$, and $f_{\text {col }}=1.6$. The outer radius of the accretion disk is $r_{\text {out }}=25 M$. $T_{\text {obs }}$ in keV; $\mathcal{F}_{\text {obs }}$ in arbitrary units and logarithmic scale. 

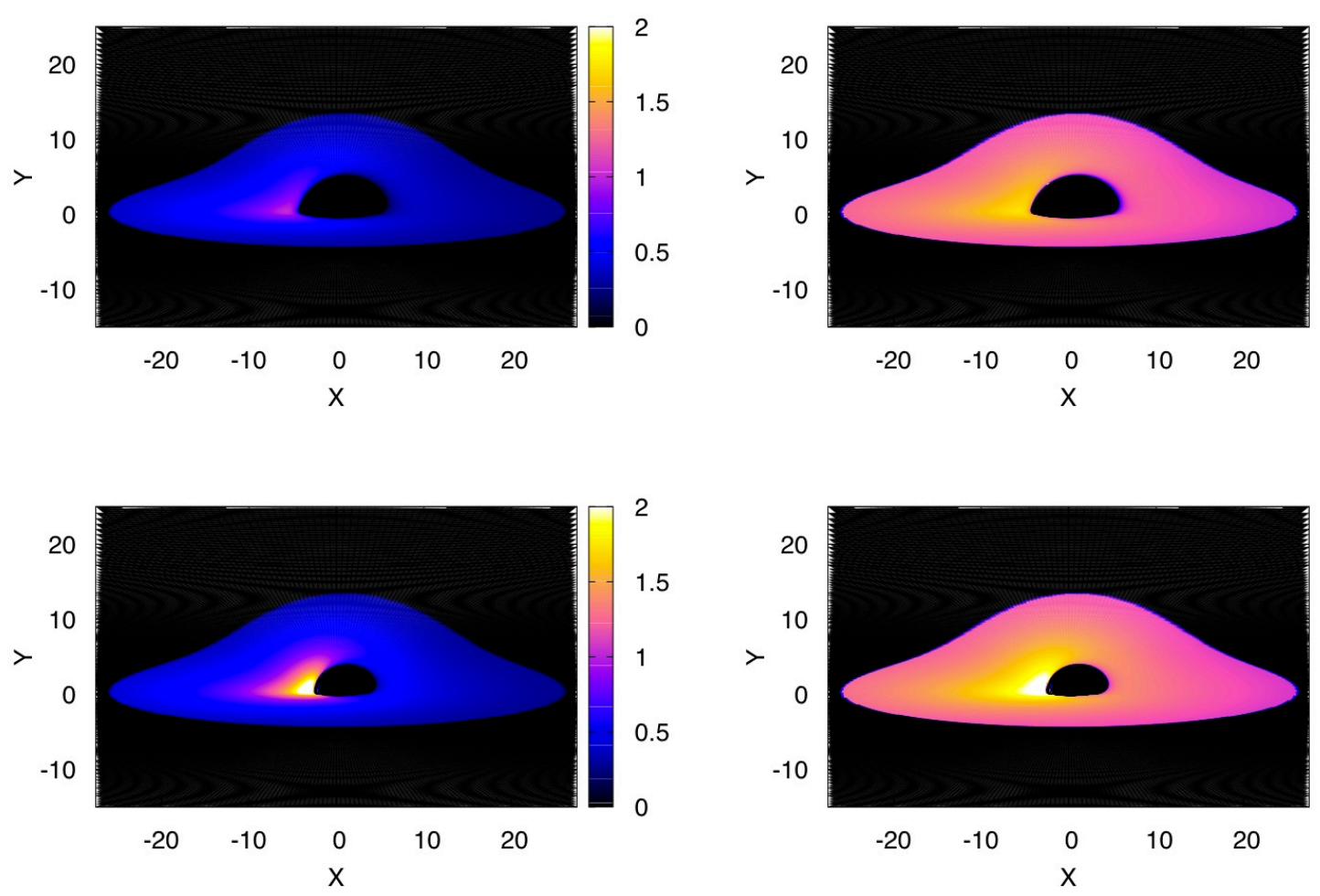

Fig. 6.- Direct image of the accretion disk. Observed blackbody temperature $T_{\text {obs }}$ (left panels) and observed flux $\mathcal{F}_{\text {obs }}$ (right panels) in Johannsen-Psaltis space-time with spin parameter $a / M=0.9$ and deformation parameter $\epsilon_{3}=-1$ (top panels) and 1 (bottom panels). The other parameters are $M=10 M_{\odot}, \dot{M}=10^{18} \mathrm{~g} \mathrm{~s}^{-1}, i=80^{\circ}$, and $f_{\text {col }}=1.6$. The outer radius of the accretion disk is $r_{\text {out }}=25 M . T_{\text {obs }}$ in $\mathrm{keV} ; \mathcal{F}_{\text {obs }}$ in arbitrary units and logarithmic scale. 


\subsection{Direct image of thin accretion disks}

In the case of a geometrically thick and optically thin accretion disk around a BH candidate, it should be possible to observe the so-called $\mathrm{BH}$ shadow; that is, a dark area over a brighter background. As the exact shape of the shadow is determined exclusively by the geometry of the space-time, its detection can be used to investigate the nature of the BH candidate (Bambi \& Freese 2009; Bambi \& Yoshida 2010; Johannsen \& Psaltis 2010; Bambi et al. 2012). In the case of geometrically thin and optically thick accretion disks, the image turns out to be quite different. For Schwarzschild and Kerr BHs, it has been already discussed in the literature (Luminet 1979; Fukue \& Yokoyama 1988; Fukue 2003; Takahashi 2004). The generalization to non-Kerr space-times is straightforward (see also Krawczynski (2012)). The temperature and the photon flux as detected by a distant observer are given by

$$
\begin{aligned}
& T_{\text {obs }}=g T_{\text {col }}, \\
& \mathcal{F}_{\text {obs }}=g^{4} \mathcal{F},
\end{aligned}
$$

where $g$ is the redshift factor given by Eq. (5). Eq. (21) follows from the definition of $g$ and from Eq. (3), while Eq. (22) is a consequence of the Liouville's theorem $I_{\mathrm{e}}\left(\nu_{\mathrm{e}}\right) / \nu_{\mathrm{e}}^{3}=$ $I_{\text {obs }}\left(\nu_{\text {obs }}\right) / \nu^{3}$. Fig. 5 shows the direct image of $T_{\text {obs }}$ and $\mathcal{F}_{\text {obs }}$ for a Schwarzcshild BH (top panels) and for a Kerr BH with $a / M=0.9$ (bottom panels), in the case of an observer with inclination angle of $80^{\circ}$. The asymmetry with respect to the $X$ axes is due to the effect of light bending, while the one with respect to the $Y$ axes comes from the Doppler boosting. Fig. 6 shows the same images in the case the accretion disk is in the JP space-time with spin $a / M=0.9$ and deformation parameter $\epsilon_{3}=-1$ (top panels) and 1 (bottom panels). As we can see, the shape of the central dark area and the intensity map of the image depend clearly on the geometry of the space-time around the compact object. Such images are definitively impossible to observe with current X-ray facilities, but they may be seen with future X-ray interferometry techniques.

\subsection{Light curve during an eclipse}

The observation of the light curve when the accretion disk around a $\mathrm{BH}$ candidate is eclipsed by the stellar companion can also provide information about the geometry of the space-time around the compact object (Fukue \& Yokoyama 1988). While current observational facilities would already have the correct time resolution to observe this feature, so far we know only one binary system with a BH candidate showing an eclipse in the X-ray spectrum. This source is M33 X-7, which is unfortunately quite far and dim, and present 
detectors cannot measure its light curve with an accuracy to observe any relativistic effect (Pietsch et al. 2004).

Fig. 7 shows the light curves of the ingress (left panel) and egress (right panel) during an eclipse for the four cases shown in Figs. 5 and 6. The inclination angle of the observer is still $i=80^{\circ}$. In these simulations, I assume that the angular momentum of the companion star is parallel to the one of the accretion disk, as it should be more likely expected from considerations on the evolution of the system. That means that the companion star occults first the approaching (blue-shifted) part of the disk and then the receding (red-shifted) one. The companion star is simply modeled as an obstacle with vertical edge and its atmosphere is completely neglected, so the disk's luminosity at the time $t$ is

$$
L(t)=\int h_{i}\left(X-v_{\mathrm{c}} t\right) I_{\mathrm{obs}}(\nu) \frac{d X d Y}{D^{2}},
$$

where $i=$ ingress or regress, $v_{\mathrm{c}}$ is the velocity of the stellar companion, and

$$
\begin{gathered}
h_{\text {ingress }}\left(X-v_{\mathrm{c}} t\right)= \begin{cases}0 & \text { if } X-v_{\mathrm{c}} t \leq 0, \\
1 & \text { if } X-v_{\mathrm{c}} t>0 .\end{cases} \\
h_{\text {egress }}\left(X-v_{\mathrm{c}} t\right)= \begin{cases}1 & \text { if } X-v_{\mathrm{c}} t<0, \\
0 & \text { if } X-v_{\mathrm{c}} t \geq 0 .\end{cases}
\end{gathered}
$$

As the shape of the light curve depends on the background metric, the possible detection of this feature can be used to constrain possible deviations from the Kerr nature of a $\mathrm{BH}$ candidate. However, in order to extract information on the space-time geometry from real data, a reliable atmospheric model would be necessary (Takahashi \& Watarai 2007).

If we could get accurate images of the disk, like the ones shown in Figs 5 and 6, we could determine at the same time $a$ and $\epsilon_{3}$. However, that is surely out of reach in the near future. In the case of the light curve during an X-ray eclipse, basically we measure the slope of the curve and the asymmetry between ingress and egress. Assuming a Kerr background, we could immediately estimate $a$ (assuming $M$ is known independently). If we are going to test the Kerr-nature of the BH candidate, we find a degeneracy between $a$ and $\epsilon_{3}$. However, the light curve is essentially sensitive only to the effect of Doppler boosting; that is, the asymmetry of the apparent image of the disk with respect to the $X=0$ axes. It is not very sensitive to the effect of light bending (responsible to the asymmetry with respect to the $Y=0$ axes) and to the gravitational redshift (which produces corrections symmetric with respect to the $X=0$ axes). The combination of the fit of the thermal spectrum and of the light curve during an eclipse for the same BH candidate should thus break the degeneracy between $a$ and $\epsilon_{3}$ and allows for the identification of a limited allowed region in the spin parameter-deformation parameter plane. 


\section{Conclusions}

General Relativity has been tested and verified in Earth's gravitational field, in the Solar System, and by studying the motion of binary pulsars. Thanks to recent theoretical progresses and high-quality data from present and near-future observational facilities, it is now possible to start testing the theory in the strong field regime. One of the most intriguing predictions of General Relativity is that the final product of the gravitational collapse is a Kerr black hole: a very simple object completely characterized by only two quantities (the mass $M$ and the spin angular momentum $J$ ) in a very specific way. The study of the thermal spectrum of geometrically thin accretion disks around stellar-mass black hole candidates can provide information about the geometry of the space-time and can thus be used to check the Kerr black hole paradigm.

In this paper, I have presented a code based on a ray-tracing approach and designed to compute the thermal emission of thin accretion disks around generic compact objects. In particular, the code can compute the thermal spectrum of a thin disk around a compact object with mass $M$, spin parameter $a$, and a deformation parameter which measures possible deviations from the Kerr background. By comparing these theoretical predictions with X-ray data of stellar-mass $\mathrm{BH}$ candidates in the high-soft state, one can constrain the nature of the compact object in the spin parameter-deformation parameter plane. Constraints from specific sources will be presented in a forthcoming paper (Bambi et al. 2013). The code can also be used to compute other observational features of a thin disk, like its direct image (Figs. 5 and 6) or its light curve during an eclipse (Fig. 7), which may be observed with future X-ray facilities.

I would like to thank Luca Maccione for fixing a bug in the code. This work was

supported by the Humboldt Foundation, Fudan University, and the Thousand Young Talents Program.

\section{REFERENCES}

Bambi, C. 2011a, Europhysics Letters, 94, 50002

Bambi, C. 2011b, JCAP 1105, 009

Bambi, C. 2011c, Modern Physics Letters A, 26, 2453

Bambi, C. 2011d, Physics Letters B, 705, 5 
Bambi, C. 2012a, Phys. Rev. D, 85, 043001

Bambi, C. 2012b, Phys. Rev. D, 85, 043002

Bambi, C. 2012c, arXiv:1204.6395

Bambi, C. 2012d, JCAP 1209, 014

Bambi, C., \& Barausse, E. 2011a, ApJ, 731, 121

Bambi, C., \& Barausse, E. 2011b, Phys. Rev. D, 84, 084034

Bambi, C., Caravelli, F., \& Modesto, L. 2012, Physics Letters B, 711, 10

Bambi, C., \& Freese, K. 2009, Phys. Rev. D, 79, 043002

Bambi, C., Guainazzi, M., \& Svoboda, J. 2013, in preparation

Bambi, C., \& Modesto, L. 2011, Physics Letters B, 706, 13

Bambi, C., \& Yoshida, N. 2010, Classical and Quantum Gravity, 27, 205006

Bertotti, B., Iess, L., \& Tortora, P. 2003, Nature, 425, 374

Carter, B. 1971, Physical Review Letters, 26, 331

Chen, S., \& Jing, J. 2012, Phys. Rev. D, 85, 124029

Everitt, C. W. F., Debra, D. B., Parkinson, B. W., et al. 2011, Physical Review Letters, 106, 221101

Fabian, A. C., Nandra, K., Reynolds, C. S., et al. 1995, MNRAS, 277, L11

Fukue, J. 2003, PASJ, 55, 155

Fukue, J., \& Yokoyama, T. 1988, PASJ, 40, 15

Hawking, S. W. 1972, Communication in Mathematical Physics, 25, 152

Johannsen, T., \& Psaltis, D. 2010, ApJ, 718, 446

Johannsen, T., \& Psaltis, D. 2011, Phys. Rev. D, 83, 124015

Johannsen, T., \& Psaltis, D. 2012, arXiv:1202.6069

Kalogera, V., \& Baym, G. 1996 ApJ, 470, L61 
Kramer, M., Stairs, I. H., Manchester, R. N., et al. 2006, Science, 314, 97

Krawczynski, H. 2012, ApJ, 754, 133

Li, L.-X., Zimmerman, E. R., Narayan, R., \& McClintock, J. E. 2005, ApJS, 157, 335

Liu, C., Chen, S., \& Jing, J. 2012, ApJ, 751, 148

Luminet, J.-P. 1979, A\&A, 75, 228

Lund, E., Bugge, L., Gavrilenko, I., et al. 2009, Journal of Instrumentation, 4, P04001

Maoz, E. 1998, ApJ, 494, L181

McClintock, J. E., Narayan, R., Davis, S. W., et al. 2011, Classical and Quantum Gravity, 28,114009

Miller, J. M. 2007 ARA\&A, 45, 441

Narayan, R. 2005, New Journal of Physics, 7, 199

Novikov, I. D., \& Thorne, K. S. 1973, in Black Holes, edited by C. De Witt and B. De Witt (Gordon and Breach, New York, US), 343

Page, D. N., \& Thorne, K. S. 1974, ApJ, 191, 499

Penrose, R. 1969, Nuovo Cimento Rivista Serie, 1, 252

Pietsch, W., Mochejska, B. J., Misanovic, Z., et al. 2004, A\&A, 413, 879

Poisson, E. 2004, A Relativist's Toolkit: The Mathematics of Black-Hole Mechanics (Cambridge University Press, Cambridge, UK)

Reynolds, C. S., \& Nowak, M. A. 2003, Phys. Rep., 377, 389

Robinson, D. C. 1975, Physical Review Letters, 34, 905

Shakura, N. I., \& Sunyaev, R. A. 1973, A\&A, 24, 337

Takahashi, R. 2004, ApJ, 611, 996

Takahashi, R., \& Watarai, K. 2007, MNRAS, 374, 1515

Weisberg, J. M., \& Taylor, J. H. 2005, ASP Conf. Ser., 328, 25

Will, C. M. 2006, Living Reviews in Relativity, 9, 3 
Williams, J. G., Turyshev, S. G., \& Boggs, D. H. 2004, Physical Review Letters, 93, 261101

Zhang, S. N., Cui, W., \& Chen, W. 1997, ApJ, 482, L155 

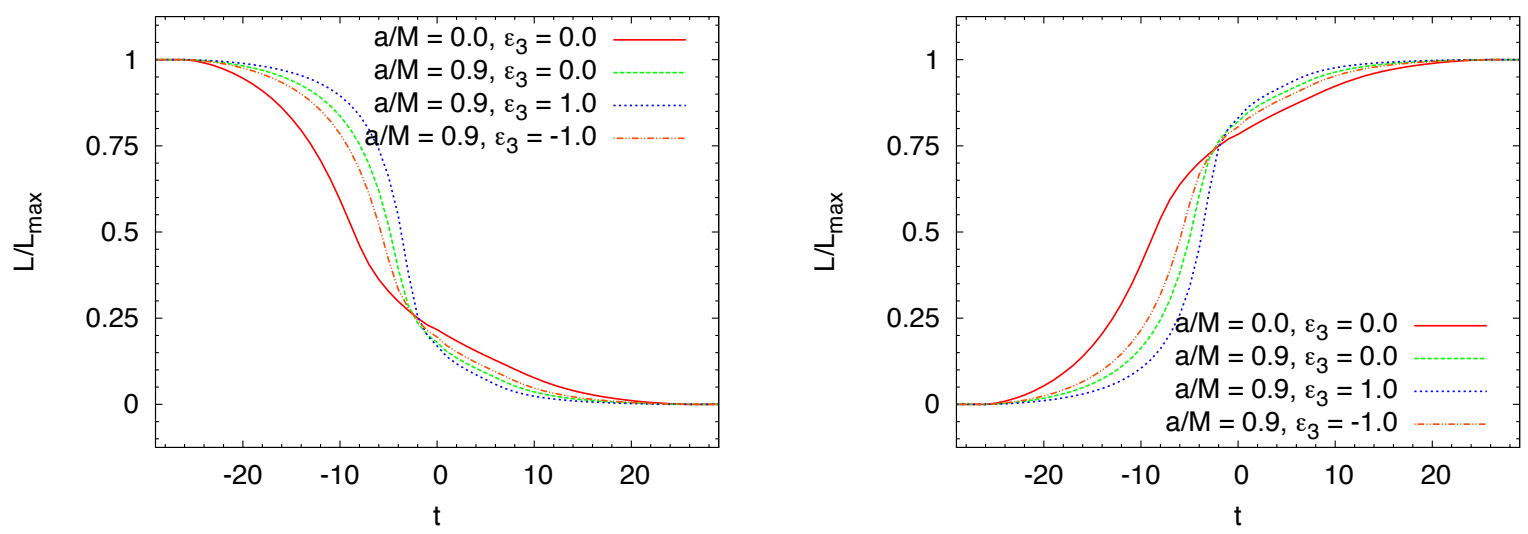

Fig. 7.- Light curves of ingress (left panel) and egress (right panel) during an eclipse for the cases of Figs. 5 and 6 . Time $t$ in units of $M / v_{\mathrm{c}}$, where $M$ is the mass of the black hole candidate and $v_{\mathrm{c}}$ is the velocity of the stellar companion. See the text for details. 